\title{
Direct measurement of pump-induced phase modulation in erbium-doped fibres
}

\author{
Thirstrup, Carsten; Shi, Yuan; Poulsen, Ove; Palsdottir, Bera
}

Published in:

Proceedings of 5th European Quantum Electronics Conference

Publication date:

1994

Document Version

Publisher's PDF, also known as Version of record

Link back to DTU Orbit

Citation $(A P A)$ :

Thirstrup, C., Shi, Y., Poulsen, O., \& Palsdottir, B. (1994). Direct measurement of pump-induced phase modulation in erbium-doped fibres. In Proceedings of 5th European Quantum Electronics Conference (pp. 198199). IEEE.

\section{General rights}

Copyright and moral rights for the publications made accessible in the public portal are retained by the authors and/or other copyright owners and it is a condition of accessing publications that users recognise and abide by the legal requirements associated with these rights.

- Users may download and print one copy of any publication from the public portal for the purpose of private study or research.

- You may not further distribute the material or use it for any profit-making activity or commercial gain

- You may freely distribute the URL identifying the publication in the public portal

If you believe that this document breaches copyright please contact us providing details, and we will remove access to the work immediately and investigate your claim. 
ing spectrum with increasing of the pumping current. Within the limits of the free-running $\mathrm{cw}$ generation regime $\left(I_{p}=\right.$ $120 \mathrm{~mA}$ ), no evidence of chirping in the spectrum is observed. The modal chirp appears when passing to the mode-locking regime $\left(I_{p}=130 \mathrm{~mA}\right)$, and its value increases with pumping current. As mode-locking develops, the laser specmode-locking develops, the laser spectrum becomes markedly asymmetric $\left(I_{p}=\right.$
$150 \mathrm{~mA})$. This effect can be explained by the fact that the dispersion of the laser active medium decreases with wavelength. At $I_{r}=150 \mathrm{~mA}$, the minimal value of time-bandwidth product $\Delta \tau \Delta v=0.52$ was registrated. It should be noted that the optical pulses obtained are not transform-limited ones due to the chirp effect and it is possible to compress them in a medium with a proper dispersion. With further increase in pumping current $\left(I_{p}=\right.$ $170 \mathrm{~mA}$ ), the lasing spectrum broadens (with the corresponding increase in the time-bandwidth product) and becomes symmetric again, which is supposedly due to gain saturation for longer wavelength modes.

An important advantage of the lasers investigated is that their entire spectrum lies in the amplification band of erbiumdoped fibers. Being combined with fiber amplifiers, these lasers could serve as source of soliton pulses with a very high repetition rate. It should be also noted that the measurement of laser characteristics was being carried out during apistics was being carried out during ap
proximately 50 hours of operation under $\mathrm{cw}$ pumping and no degradation of spectra or pulse parameters was observed.

$\mathrm{CW}$ passive mode-locking was observed for the first time in InGaAsP laser diodes with saturable absorber regions created by the ion implantation. Optical pulses of $650 \mathrm{fs}$ width at $110-\mathrm{GHz}$ repetition rate were obtained in the spectral range of $1.53-1.54 \mu \mathrm{m}$. No changes in spectra and autocorrelation traces were observed after 50 hours of cw operation. *Institute of Physics and Technology Russian
Academy of Sciences, 26 Politekhnicheskaya

str., St. Petersburg 194021

1. Y. K. Chen, M. C. Wu, T. Tanbun-Ek R. A. Logan, M. A. Chin, Appl. Phys. Lett. 58(12), 1253-1255 (1991).

2. K. Y. Lau, IEEE J. of Quant. El

3. J. H. Zarrabi, E. L. Portnoi, A. V. Chelnokov, Appl. Phys. Lett., 15261528 (1991). 26(2), 250-261 (1990).
QThF

1100

Rooms T \& U

Special Nonlinear Materials

Jo Zyss, CNET, France, Presider

QThF1

1100

Dynamics and origin of the optical Kerr effect in CdSe nanoparticles in the resonant regime

M. Ghanassi, M. C. Schanne-Klein, D.

Ricard, C. Flytzanis, Laboratoire d'Optique Quantique du C.N.R.S., Ecole Polytechnique 91128 Palaiseau Cedex, France

In the resonant regime, the optical Kerr effect of CdSe-doped glasses is due to photoexcitation of carriers whose pres ence modifies the optical properties of the CdSe quantum dots. The recovery time, which governs the repetition rate at which such devices could be operated, is equal to the recombination time of these carriers. At low laser intensity, the recom bination time may be shortened by the photodarkening effect.' At higher intensity, nonlinear processes such as Auger recombination may play a significant role as aleady suggested ${ }^{2}$ although the first data could not clearly make the differ ence between a cubic (Auger) process or a quadratic one

Using three different time-resolved techniques, optical phase conjugation, nonlinear absorption, and time-resolved luminescence, we clearly observed that for a fresh sample, at high laser intensity and for not too long delay times, carrie recombination is dominated by Auger processes. All these data lead to an Auger constant on the order of a few $10^{-30} \mathrm{~cm}^{6}$ $\mathrm{s}^{-1}$. When the sample has been photodarkened, both the linear nonradiative decay and the Auger process are important.

Understanding the origin of the nonlinearity in these media is also of importance since such artificial materials can, in principle, be tailored to meet specific requirements. The optical Kerr effect may be due to free carriers in which case it corresponds to saturation of the first electronic transition and to induced absorption between the corresponding one-pair state and two-pair states. Alternatively, the photoexcited carriers may be trapped at the surface of the nanoparticles and, through the static electric field they create for example, they may also modify the optical properties.

Time resolving the nonlinear response at low laser intensity and in the degenerate case for samples having experienced various degrees of photodarkening, we have been able to access the relevant mechanisms. Usually, the response shows both a fast component due trapped carriers. The larger the darkentrapped carriers. The larger the darkening dose is, the smaller the time constant of the fast component and the smaller the magnitude of the slow component. The dominant mechanism is thus observed to depend on the origin of the sample and nonlinear response is the most reliable technique of determining it.

1. P. Roussignol, D. Ricard, J. Lukasik, C. Flytzanis, J. Opt. Soc. Am. B 4, 5 (1987).

2. P. Roussignol, M. Kull, D. Ricard, F. de Rougemont, R. Frey, C. Flytzanis, Appl. Phys. Lett. 51, 1882 (1987) and 54, 1705 (1989).

QThF2 1115

Direct measurement of pump-induced phase modulation in erbium-doped fibres

Carsten Thirstrup, Yuan Shi, Ove Poulsen, Bera Pálsdóttir," Mikroelektronik Centret,

Technical University of Denmark, Building

345E, 2800 Lyngby, Denmark

A system, which is able to determine pump induced phase modulation in $\mathrm{Er}$ doped fibres for phase shifts $\phi_{\mathrm{p}}$ ranging from $\sim \pi / 20$ to several $\pi$, has been developed. The system (see Fig. 1) consists of a Mach-Zehnder interferometer using a polarization maintaining $3-\mathrm{dB}$ fibre coupler. One arm of the interferometer includes an Er-doped fibre pumped by a 980-nm Ti:sapphire laser. Light from a tunable semiconductor signal laser $\left(\lambda_{\mathrm{s}}=\right.$ $1.5 \mu \mathrm{m}-1.6 \mu \mathrm{m})$ is coupled to both interferometric arms. A differential detection scheme improves the sensitivity and eliminates influence of amplified spontaneous emission. The phase in the reference arm is linearly modulated in time, $\phi_{m}=\omega t$, by means of a piezo-electric crystal. This results in a response of the detected signal, which in the case of no pump power can be written;

$$
\Delta \mathrm{I}_{\mathrm{det}} \propto \cos \left(\omega \mathrm{t}+\phi_{0}\right)
$$

and in presence of pump power;

$$
\Delta \mathrm{I}_{\text {det }} \propto \cos \left(\omega \mathrm{t}+\phi_{0}+\phi_{\mathrm{p}}\right),
$$

where $\phi_{0}$ is an arbitrary phase and $\phi_{\mathrm{P}}$ is the pump-induced phase shift of the signal. $\phi_{p}$ is determined by measuring the phase difference of the cosine waveforms before and after the pump is turned on by an optical chopper.

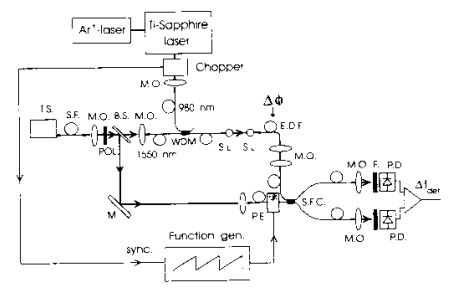

QThF2 Fig. 1. Schematic of the setup for directly measuring pump-induced phase modulation in Er-doped fibres. T.S.: tunable semiconductor laser; S.F.: single mode fibre; M.O.: microscope objective; POL.: polarizer; B.S.: beam splitter; M.: mirror; WDM: $980 \mathrm{~nm} / 1550$ nm wavelength demultiplexer; S.L. spherical lens; E.D.F.: Er-doped fibre; P.E.: piezo-electric crystal; S.F.C.: (polarization maintaining) 3-dB single mode fibre coupler; F.: pump block filter; P.D.: photodiode. 


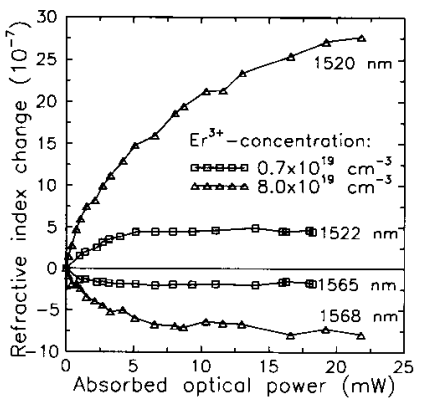

QThF2 Fig. 2. Refractive index change as function of wavelength for Er-doped fibres with a doping concentration of $0.7 \times 10^{19} \mathrm{~cm}^{-3}$ (square symbols), $2 \times 10^{19} \mathrm{~cm}^{-3}$ (cross symbols) and $8 \times 10^{19} \mathrm{~cm}^{-3}$ (triangular symbols). The pump power at $980 \mathrm{~nm}$ is $4 \mathrm{~mW}$.

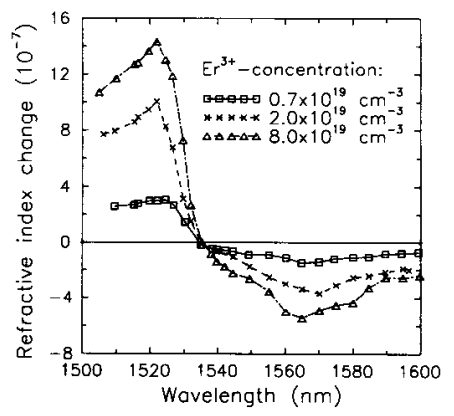

QThF2 Fig. 3. Refractive index change as function of absorbed pump power for Er-doped fibers with doping concentrations of $0.7 \times 10^{19} \mathrm{~cm}^{-3}$ (square symbols) and $8 \times 10^{19} \mathrm{~cm}^{-3}$ (triangular symbols) at different wavelengths as indicated.

In Fig. 2, the change in refractive index defined as, $\Delta \mathrm{n}=\left(\lambda_{\mathrm{s}} /(2 \pi \mathrm{L}) \phi_{\mathrm{p}}\right.$, where $\mathrm{L}$ is the length of the fibre, is plotted for three fibres with $\mathrm{Er}^{3+}$-doping concentrations of $0.7 \times 10^{19} \mathrm{~cm}^{-3}$ (square symbols) $2 \times 10^{19} \mathrm{~cm}^{-3}$ (cross symbol) and $8 \times 10^{19}$ $\mathrm{cm}^{-3}$ (triangular symbols) as function of wavelength at an absorbed pump power of $4 \mathrm{~mW}$. The $\Delta n$ increases with increasing doping concentration and changes sign at $\lambda_{s}=1535 \mathrm{~nm}$, which is situated just below a resonance wavelength of $1530 \mathrm{~nm}$. The slope of the curves and therefore the dispersion are maximum near resonance. The dispersion for the fibre with the highest doping concentration is calculated to be $\sim 200 \mathrm{ps} / \mathrm{nm} / \mathrm{km}$ at $1530 \mathrm{~nm}$.

Figure 3 shows $\Delta \mathrm{n}$ as function of absorbed pump power for the fibres with $\mathrm{Er}^{3+}$-concentrations of $0.7 \times 10^{19} \mathrm{~cm}^{-3}$ (square symbols) and $8 \times 10^{19} \mathrm{~cm}^{-3}$ (triangular symbols). The wavelengths depicted in Fig. 3, $\lambda_{\mathrm{s}}=1565 \mathrm{~nm}$ and 1568 $\mathrm{nm}$ are situated below resonance and the wavelengths $\lambda_{s}=1522 \mathrm{~nm}$ and $1520 \mathrm{~nm}$ are situated above resonance, where a larger $\Delta n$ can be obtained. The $\Delta n$ satu- rates at higher pump powers for the fibre with the higher doping concentration. Thermal effects are believed to be negligible, since a thermal contribution to $\Delta \mathrm{n}$ should be either positive or negative and independent of wavelength, in contrary to the results shown in Fig. 3.

The proposed system has proven to be useful to measure pump-induced phase shifts directly and refractive index changes in Er-doped fibres. The results show that a highly doped fibre should be used if refractive index modulation is desirable (e.g., in optical switches). In applications where the dispersion is unwanted (e.g., in soliton transmission systems), a tradeoff between gain and refractive index modulation should be made for the operating wavelength. ${ }^{*}$ Lycom A/S, NKT Alle 75, 2605 Brondby, Denmark

\section{QThF3 (Invited)}

1130

Two-photon absorption of semiconductor crystallites in doped glasses

G. P. Banfi, V. Degiorgio, M. Ghigliazza, H. M. Tan," A. Tomaselli, Dipartimiento d Elettronica, Universitá di Pavia, Via Abbiategrasso 209, Pavia, Italy

One of the questions concerning semiconductor doped glasses (SDG) is whether the nonlinearities of the crystallites at frequencies well below bandgap are affected by the electronic confinement. The problem has both conceptual and practical implications. Large effects and practical implications. Large effects even for modest confinement have been suggested by theoretical models, but the data so far reported are not conclusive to this regard. ${ }^{1-2}$ We here report the first quantitative determination of $I m \chi_{m}$, the imaginary part of the third order suscep-
tibility of the crystallites in SDG. The ten tibility of the crystallites in SDG. The ten
samples investigated are commercial samples investigated are commercial
SDG manufactured by Schott, and contain CdTe (RG850-RG830) or CdSSe (CG-495 to RG 715) crystallites.

In order to obtain $I m \chi^{(3)}$, one first derives, through a nonlinear transmission measurement, $\beta_{S D C}$, the two-photon absorption (TPA) coefficient of the SDC $\left(\beta_{\mathrm{SDG}}\right.$ is related to $\operatorname{Im} \chi_{\mathrm{SDC}}^{(3)}$ through $\beta=$ $\left(\omega\left(\epsilon_{0} c^{2} n^{2}\right)^{-1} I m \gamma^{(3)}\right)$ and then makes use of the relation $\chi_{n n}^{(3)}=\chi_{S D C}^{(3)} f^{4} f_{v v}$ with $\mathrm{f}$ the local field correction factor, and $f_{\mathrm{y}}$ the volume fraction occupied by the crystallites. ${ }^{3}$ The crucial quantities in the procedure are $\beta_{\text {sDC }}$ and $f_{w}$

Since the volume fraction cannot be obtained neither from a wet analysis, nor from the initial composition of the SDG melt this problem was solved by using small angle neuron scattering measurements (performed at the Cold Neutron facility at NIST, Gaithersburg, Maryland) which provided accurate values of $f_{v}$ and of the average radius $R$ of the crystallites for each sample. ${ }^{4}$ For our samples, $f_{\mathrm{v}}=$ $1.5-5 \%, \mathrm{R}=2-11 \mathrm{~nm}$.

Some transmission data are shown in Fig. 1. In order to obtain a reliable $\beta_{\text {spc }}$ from an otherwise simple transmission measurement one should take into account the effect of collisional absorption. In fact, the optical pulse is attenuated not only by TPA, but also by the carriers excited by TPA. In the measurements at $1.06 \mu \mathrm{m}$ (were we employed $30 \mathrm{ps}$

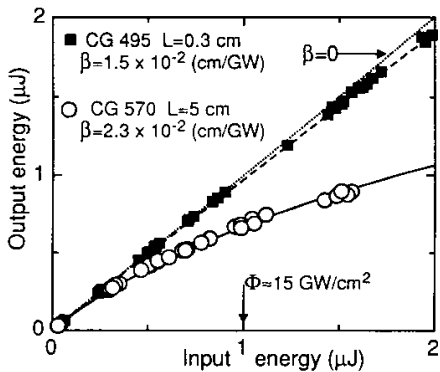

QThF3 Fig. 1. Output energy versus input energy and best fit (solid curves). Femtosecond data at $605 \mathrm{~nm}$.

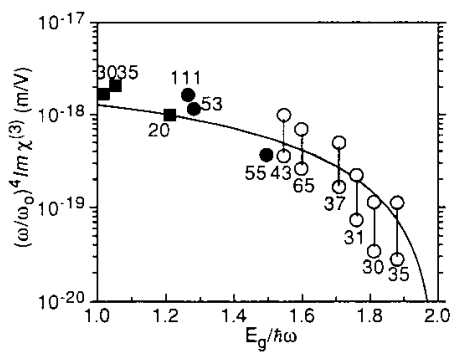

QThF3 Fig. 2. Plot of the scaled quantity $\left(\omega / \omega_{0}\right)^{4} \operatorname{Im} \chi^{(3)}$ versus $E_{g}(\hbar \omega)$. Optical measurements at: $0.6 \mu \mathrm{m}$ (squares), $1.06 \mu \mathrm{m}$ (circles). The uncertainty for glasses with $\mathrm{E}_{\mathrm{g}} /(\hbar \omega)>$ stems from the indeterminacy in collision absorption: the two open circles encompass the possible extreme values. The numbers associated to the experimental points are the radii of the nanocrystals in angstroms. The solid line gives $\left(\omega / \omega ;{ }^{\prime} ;\right)^{4} I m \chi^{(3)}$ for bulk semiconductors.

pulses), we could account for collisional absorption, at least for three glasses, by comparing results obtained with samples of different thickness. Another possibility is to employ much shorter pulses, which generate a small number of free carriers. Measurement were done at LENS, Florence, with $200 \mathrm{fs}$ pulses at $\lambda=0.6 \mu \mathrm{m}$. The available wavelength allowed only the study of $O G 495,570,590$.

The results can be seen in Fig. 2 where we plotted the scaled quantity ( $\omega$ / $\left.\omega_{0}\right)^{4} I m x^{(3)}$, with $\omega_{0}$ the frequency of 1.06 $\mu \mathrm{m}$ radiation, as a function of $\mathrm{Eg}(\hbar \omega)$ with $E_{g}$ the energy gap and $\hbar \omega$ the photon energy. The solid line gives the the oretical value of $\left(\omega / \omega_{0}\right)^{4} \operatorname{Im} \chi^{(3)}$ for a bulk semiconductor, and, as indicated by Van Stryland, ${ }^{5}$ is in agreement with bulk data. Im $x^{(3)}$ must be quite small, if we note that deviations from the bulk values are not evident even for the small crystallites of the GG 495 glass $(R=2 \mathrm{~nm})$. We can conclude that semiconductor nanocrystals with a size ranging between 2 and $11 \mathrm{~nm}$ the TPA coefficient is comparable to that the bulk semiconductor in the whole accessible range of $E_{g} /(\hbar \omega)$ values. Such a result calls for a reconsideration of the 